\title{
Researcher of the Year 2020 Professor Herbert Gleiter, Germany
}

\author{
Ashutosh Tiwari* iD \\ Secretary General, International Association of Advanced Materials, Gammalkilsvägen 18, Ulrika 590 53, Sweden \\ *Corresponding author: E-mail: secretarygeneral @iaamonline.org; Tel: (+46) 1313-2424 \\ DOI: $10.5185 /$ amlett.2020.061520
}

Researcher of the year is considered one of the highest honors for a researcher working in a diverse background, either from Physical, Chemical, Biological, Engineering, Mathematical and Medical Sciences along with their substantial contribution towards 'research perspective, expanding education, promoting materials research and its applications at a global standard', mentoring professionals and series of advancements on the related topics.

International Association of Advanced Materials, IAAM had the distinct pleasure of honouring Professor Herbert Gleiter with the prestigious title of 'Advanced Materials Laureate' during the $30^{\text {th }}$ IAAM Felicitation ceremony held at the Asian Advanced Materials Congress, Singapore in November 2019 (Fig. 1) [1,2]. It is one of the highest honours that International Association of Advanced Materials, IAAM confers upon its delegates and members for their substantial contributions towards the advancements of materials to global excellence [3]. Moreover, it is one of the biggest honours that a researcher working in the sphere of advanced materials can receive.

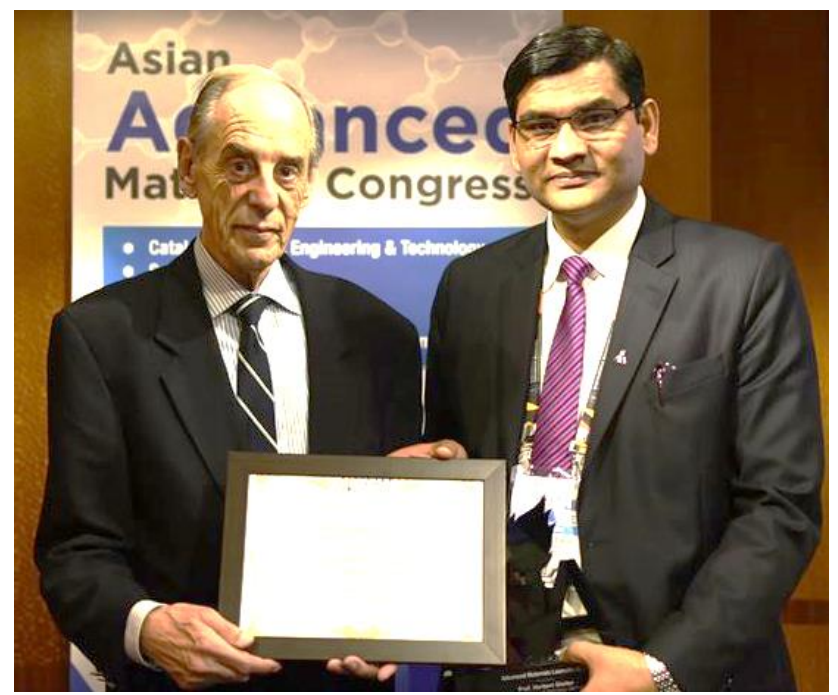

Fig. 1. Prof. Herbert Gleiter with the Advanced Materials Laureate during the $30^{\text {st }}$ IAAM Award Assembly, Singapore.

Likewise, it is a core belief of the International Association of Advanced Materials, IAAM that recognition and appreciation of the scientific works are important and critical for the advancement of the scientific community [4]. In line with this belief, every year, International Association of Advanced Materials, IAAM confers upon the deserving members of the scientific community with several awards and recognitions. The highest of these recognitions is the title of 'Researcher of the Year' which is recognition of the outstanding contributions and achievements of a leading researcher towards the 'advancement of materials for global excellence' (Fig. 2).

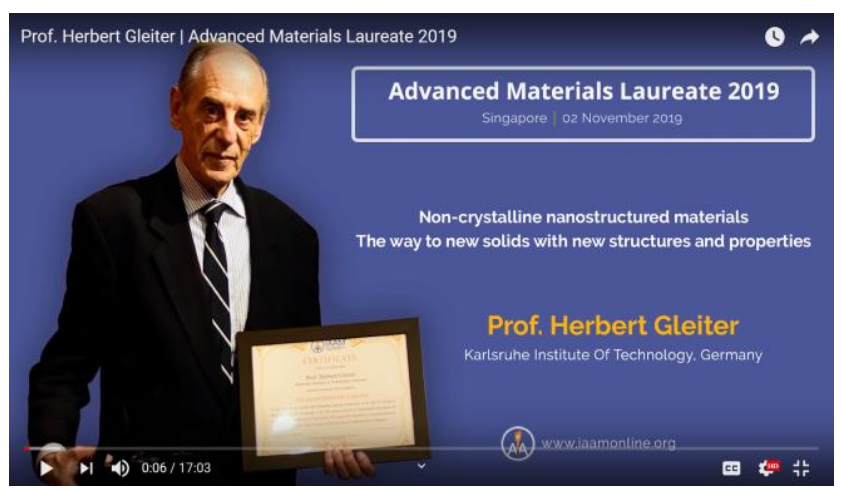

Fig. 2. Interview and the Advanced Materials Laureate Lecture of Professor Herbert Gleiter with IAAM media at Asian Advanced Materials Congress 2019, Singapore.

https://www.youtube.com/watch?v=rxdYgcABolQ

\section{Advanced Materials Laureate Lecture}

Prof. Gleiter had delivered Advanced Material Laureate Lecture in Singapore on the overview of "non-crystalline nanostructured materials- the way to new solids with new structures and properties" [1]. In his laureate lecture, Prof. Gleiter had summarized that, "The technologies being used today are primarily based on utilizing crystalline materials (e.g. metals, semiconductors, or crystalline ceramics). The path to new technologies may be opened by nanostructured materials that are totally or even partially non-crystalline. One such group of nanostructured materials is nanoglasses. These materials consist of nanometer-sized glassy regions connected by nanometer-wide interfacial regions with electronic and atomic structures that are not found in melt-cooled glasses. Due to these new electronic/atomic structures, the properties of nano-glass differ from the corresponding properties of melt-cooled glasses. For instance, their biocompatibility, their ductility, their catalytic, and ferromagnetic properties are changed by up to several orders of magnitude. Also, they allow the alloying of different components; e.g. ionic materials 


\section{Advanced Materials Letters www. vbripress.com/aml}

(e.g. $\mathrm{SiO}$ ) with metallic materials (e.g. PdSi glasses) that are immiscible and cannot be alloyed in the crystalline state. The properties of these nano-glasses may be controlled by varying the sizes, chemical compositions of the glassy clusters which opens the prospect of a new age of technologies, a "glass age". Another group of nanostructured partially non-crystalline materials with tunable properties are nano-porous metals with electrolyte filled pores. By applying an external voltage between the electrolyte and the nano-porous metal, their properties, e.g. their superconductivity, electric resistivity, and magnetic moment may be varied. Multi atom or switchable contacts form a third group of these kinds of materials. His 'Advanced Materials Laureate Lecture' is available at: https://www.youtube.com/watch?v=rxdYgcABolQ.

\section{Biography and research journey}

Prof. Gleiter is considered as a revolutionary researcher of the world of nanomaterials due to pioneer in nanomaterials technology. During last 30 years his ground-breaking work in nanoscience and nanotechnology has long-lasting effect on scientific enquiries. His research milestones reflected in popularity of his research and findings, which justified an influential testament to his far-reaching impact on nanomaterials world.

Prof. Herbert Gleiter was born on October 13, 1938, in Stuttgart, Germany. He studied mechanical engineering and Physics at the Technical University of Stuttgart and obtained the degree of a graduate engineer. In the year 1966, he obtained his $\mathrm{PhD}$ in Physics from the University of Stuttgart, Germany [5]. After completing his doctorate, Prof. Gleiter moved to Harvard University to conduct his post-doctorate research. In the year 1970, he obtained his D.Sc. degree in Material Science from the University of Bochum, Germany. One year later, in the year 1971, he joined the Massachusetts Institute of Technology (MIT), USA, and served as a visiting professor [6].

Professor Gleiter returned to Germany in the year 1972 and joined the Institute of Materials Science, University of Bochum, as a professor. In the year 1973, he joined the Institute of Materials Science, University of the Saarland, Germany, and went on to serve as a Professor and Director there until the year 1994. During the early 1980s, he also got calls from the University of Hamburg-Harburg, Germany and the Federal Institute of Technology, Zurich, Switzerland. The foundation of the Institute of New Materials, Germany, was laid under the direction of Prof. Gleiter in the year 1987. He served there in the capacity of a senior member and the Founding Director from 1987 to 1994. In the year 1994, the government of Germany appointed him as a member of the Executive Board of the Research Center, Karlsruhe, Germany. Four years later, he, along with Nobel Laureate J.M. Lehn and D. Fenske, he initiated the Institute of Nanotechnology at the Research Center Karlsruhe that has grown to become Germany's largest research institute in nanotechnology. He served as the Executive Director of the Institute of Nanotechnology from 1998-2004.

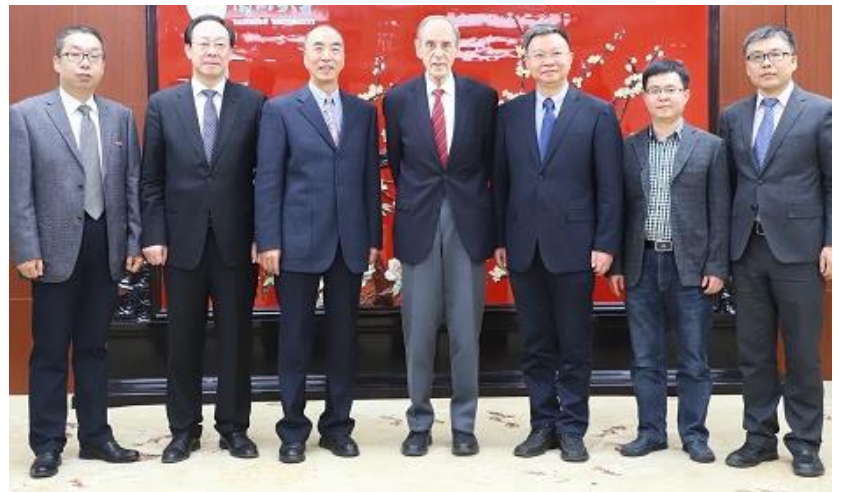

Fig. 3. Prof. Herbert Gleiter with some of the materials scientists. From left to right: Prof. Guojun Wu, Prof. Yumin LI, Prof. Jiangong LI, Prof. Herbert Gleiter, Prof. Chunhua Yan (President of Lanzhou University), Prof. Xiang Liu and Prof. Gang Chi.

Dr. Gleiter went on to serve as the Kuang-Pui Chair Professor at Zhejiang University, Hangzhou, China (20042007). He has been a Distinguished Scholar of the Korean Research Foundation (2005) and a Distinguished Professor, Arizona State University, USA (2007-2010).

In addition to this, Dr. Gleiter has been a Professor and Distinguished Senior Fellow of the Institute of Nanotechnology, Karlsruhe Institute of Technology (KIT), Germany, since 2006. He is also the Founding Director of the Herbert Gleiter Institute of Nanoscience, Nanjing University of Science and Technology (2012) and Senior Fellow of the Institute of Advanced Studies, City University of Hong Kong since 2015.

Moreover, Prof. Gleiter has also occupied major positions and membership of various scientific committees and societies from time to time (Fig. 3). He served as a member of the Scientific Committee of the Polish Academy of Sciences (1989-2003), Member of the Central Selection Committee and Awards Committee of the Alexander von Humboldt Foundation, Germany (1989-2005), Member of the Supervisory Board of BESSY, Germany (1994-1998), Member of the Supervisory Board of Forschungszentrum Jülich, Germany (1994-2000), Member of the Supervisory Board of the Hahn Schickard Society, Germany (19952000), Member of the Scientific Council of the Republic of Slovenia (2000-2003), and many more committees and panels of extreme importance and prestige. Besides all this, Dr. Gleiter has also been the Vice President of the German National Academy of Sciences Leopoldina (2007-2012), Scientific Advisor to the German Israel Foundation (19902005), and Scientific Advisor to the Hahn Meitner Institute Berlin, Germany (1994-1998) [5].

\section{Efforts for promotion of Advanced Materials}

Prof. Gleiter believes that progress in materials science is directly linked to human welfare and development of superior materials with the use of advances science and technology should be the primary goal of materials researcher across world.

Prof. Gleiter is considered as the pioneer of the nanocrystalline materials (Fig. 4). During his work at Harvard 


\section{Advanced Materials Letters www. vbripress.com/aml}

and MIT he discovered the existence of dislocations in inter-crystalline interfaces and proposed the "structural unit model" of grain boundaries which provides the basis for today's grain boundary models. Moreover, during that time he pioneered - in cooperation with M. Ashby and B. Chalmers - the application of Molecular Dynamics methods to investigate the atomic structure of intercrystalline interfaces. This work led to the proposal of the so called "structural unit model" of grain boundaries. This model provides the basis for all of today's atomistic models of inter-crystalline interfaces. In the subsequent years, this model was successfully tested by several experimental methods (e.g. electron microscopy, energy measurements by development of the "rotating sphere" method, diffusion and segregation measurements etc.).

In the subsequent years, Herbert Gleiter and his group have introduced two new concepts that turned out to be fundamental for the development Materials Science during the following decades. There, in the late 1970s, he pioneered the new class of materials: nano-crystalline materials. The idea of these new materials was to create solids consisting of a large ( $50 \%$ or more) volume fraction of inter-crystalline boundaries between crystallites with the same or with different chemical compositions (single phase or multi-phase nano-crystalline materials). Nano crystalline materials were expected to open the way to solids with new atomic structures and hence new properties.

This idea was confirmed by the rapidly growing number of subsequent studies on nano-materials worldwide Today more than 800 papers are published in this area every year, several international conferences are organized annually and most (national as well as international) conferences in the area of Materials Science have one or several sessions on "nano-materials". According to a recent study of the German Government, the annual value of the products based on nanostructured materials is already now beyond 2 billion US\$ with a growth rate of about 15 to $20 \%$ per year.

More recently, Herbert Gleiter's work focuses on the following two new areas: the first area pioneers a new concept of non-crystalline materials, called nano-glasses. Nano-glasses differ structurally in the following way from all of today's glassy materials. Contrary to today's glasses with a homogeneous microstructure, nano-glasses consist of nanometer-sized glassy regions connected by glass/glass interfaces with new non-crystalline structures (different from the structure of the glassy regions). By varying the sizes and/or the chemical compositions of the glassy regions, the volume fraction of these interfaces and hence the atomic structures and therefore also the properties of nano-glasses were varied in (partially spectacular ways), just like in crystalline materials (metals, semi-conductors, ceramics etc.) the properties of which may be changed by varying the sizes and/or chemical compositions of the crystallites. For example, FeSc nano-glasses were revealed to be strong ferromagnets although melt-quenched glasses (with the same chemical compositions) were paramagnetic. Similar variations were noted for other properties as well, e.g. the ductility, the biocompatibility, the catalytic properties of nano-glasses were improved by one or several orders of magnitude in comparison to the corresponding properties of today's glasses with comparable chemical compositions.

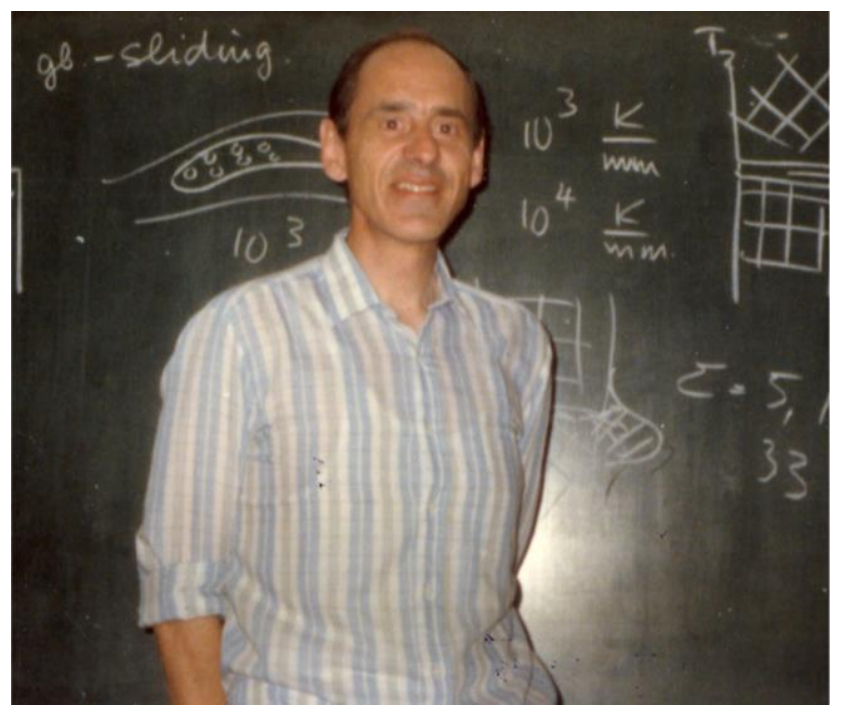

Fig. 4. Prof. Herbert Gleiter in the Deutsche Museum at Munich during exhibition for inventing the nano-crystalline materials. The photo was taken when he introduced this idea.

In fact, nano-glasses have been shown to lead to noncrystalline materials with a microstructure that corresponds to that of single or multi-phase poly-crystalline materials such as steels, ceramics etc. that provide the basis of today's technology. In other words, nano-glasses are likely to open the door to a new age of technologies based on glassy materials with a comparable wide spectrum of properties, as the crystalline materials that are available today and which provide the basis of today's technologies. An international advisory board of the Chinese Academy of Sciences (CAS) selected nano-crystalline materials and nano-glasses to be the most promising development in modern materials opening the way to a new period of technologies. Based on this evaluation it was recommended to start up a new research institute (similar to a Max Planck Institute in Germany) working on nanoscience at Nanjing University with the name of "Herbert Gleiter Institute of Nanoscience". In addition to Professor Gleiter, the directors of this institute are Profs. Ke Lu and J. Xin (from the CAS). The partners from Germany are at present Profs. H. Fuchs, H. Hahn and Schimmel.

The second field of Herbert Gleiter's present activities is the application of methods that were initially developed in nanotechnology (such as the design of new cluster beam sources, cluster detectors etc.) to probe the applicability limits of Quantum Physics to systems of macroscopic size. In these studies, Poisson's Spot experiments are performed with specimens of macroscopic sizes such nanometer-sized clusters. Theoretical studies indicate that these experiments should permit to probe the application limits of Quantum Physics to objects with a size of up to 20 million atomic 


\section{Advanced Materials Letters www. vbripress.com/aml}

mass units i.e. to objects with a size of a few nanometers such as viruses, clusters comprising many thousands of atoms etc. These experiments are expected to be of fundamental scientific significance, because they are likely to permit to answer the following fundamental question: Does Quantum Physics apply to systems of all sizes ranging from elementary particles all the way to systems of macroscopic dimensions such as grains of sand or is there a limiting size beyond which Classical Physics is the appropriate way to represent the reality.

If the Quantum Physics would be found to apply to systems of all sizes, it would mean that the macroscopic world does not exist objectively but is the result of the entanglement of the wave functions of the objects considered with the wave functions of their environment. In other words, we can never comprehend the structure and nature of any object by looking at it. All we can do is to reconstruct the nature of the object before we looked at it by means of Quantum Physics- from what we have seen by looking at the object. On the other hand, if Quantum Physics is limited to objects below a certain size, the Poisson Spot experiments seem to be able to discover that size and one could then try to find out what controls this size i.e. the limit between Classical and Quantum Physics. If such a limit would exist, it would be of fundamental importance for our understanding of the world.

In that sense, the long-range perspective of the Quantum Physics experiments performed by Herbert Gleiter and his group seem be that they open the following new perspective of nanoscience and nanotechnology. The new perspective is to utilize methods developed in nanoscience and nanotechnology as a tool for performing new kinds of studies in other areas of science. Developments of that kind appear to be similar to - for example - the application of NMR (developed in nuclear physics) in medicine or of the decay of radioactive isotopes to measure the age of archaeological objects.

\section{Highlighted scientific contributions}

His research influenced the rise of nanomaterials research around the world and helped in the development of nanoscience and nanotechnology. Today more than 800 papers are published in nanomaterials every year, several international conferences are organized annually in the area of Materials Science have one or several sessions on "nanomaterials". Prof. Gleiter and group published their finding of nanocrystalline ceramics with retained properties of ceramics via increasing a few micrometer of the material, allowing low temperature plasticity property in Nature [7]. In 1989 he initiated the development of new class of noncrystalline solids, nano-glasses and described the structure and performance of these in his work [8]. Prof. H. Gleiter has delivered high quality research in the field of the atomic or unit structure of grain boundaries and development of nanostructured materials. These materials consist of nanometer-sized crystallites with different chemical compositions and crystallographic orientations having different properties from single crystals or poly crystal of normal composition. The synthesis, characterization and processing of these nanostructured materials revolutionised the field of nanotechnology. He also published a number review article to describe the concept behind this [9].

Dr. Gleiter and team proved that the reversible strain amplitudes comparable to those of commercial piezoceramics can be induced in metals by introducing a continuous network of nanometersized pores with a high surface area and by controlling the surface electronic charge density through an applied potential relative to an electrolyte impregnating the pores. The voltage-induced reversible strain in a nanoporous metal exhibited an amplitude in excess of $0.1 \%$ analogous to previous findings with other materials and could be used as actuators [10]. Fig. 5(a) shows the scanning electron micrograph of a fracture surface, which illustrates the bicontinuous structure, consisting of an interconnected network of nanometer-sized Pt crystallites and an interconnected pore space. Fig. 5(b) is a schematic illustration of the induced charge in the electrochemical double layer at the surface of the particles. Fig. 5(c-f) shows the results of in situ cyclic scans of $E$ in the dilatometer at different scan rates, using $\mathrm{KOH}(1 \mathrm{M})$ as the electrolyte.
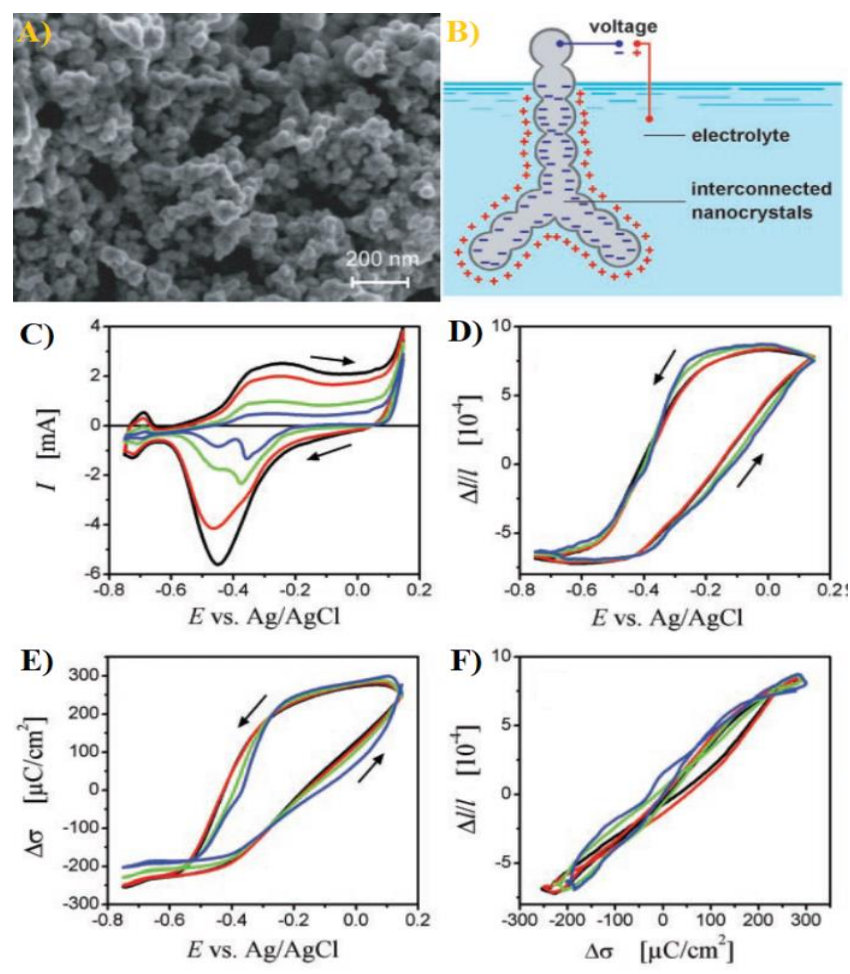

Fig. 5. (A) Scanning electron micrograph showing the fracture surface of a nanoporous Pt sample. (B) Schematic representation of an interconnected array of charged nanoparticles immersed in an electrolyte. Results of in situ cyclic scans of $E$ in the dilatometer at different scan rates, using $\mathrm{KOH}(1 \mathrm{M})$ as the electrolyte. Each curve shows the third out of three successive scans. (A) Charging current $I$ versus $E$. (B) Relative length change $\Delta l / l$ versus $E$. (C) Change $\Delta \sigma$ in the specific surface charge density versus $E$. (D) $\Delta l / l$ versus $\Delta \sigma$. Arbitrary points in the center of the intervals of experimental values were chosen for the zero of the strain and charge density. Scan rates were $1 \mathrm{mV} / \mathrm{s}$ (black), $0.8 \mathrm{mV} / \mathrm{s}$ (red), $0.4 \mathrm{mV} / \mathrm{s}$ (green), $0.2 \mathrm{mV} / \mathrm{s}$ (blue). Arrows denote the direction of the scan (Reproduced with permission from [10], Copyright (C) 2003, The American Association for the Advancement of Science). 


\section{Advanced Materials Letters www. vbripress.com/aml}

Recently his team showed the $\mathrm{Fe}_{90} \mathrm{Sc}_{10}$ nanoglass system to have enhanced magnetic properties [11]. The nanoglass prepared by cold compaction of glassy nanospheres has ferromagnetic property and the isolated nanospheres were paramagnetic in nature (Fig. 6). The report describes that a nanoglass with magnetic (and probably other density-related) properties differing significantly from those of the conventional amorphous counterpart with identical chemical composition, i.e., a glass produced by melt-spinning. This is considered as the first of a kind of literature in the materials science.
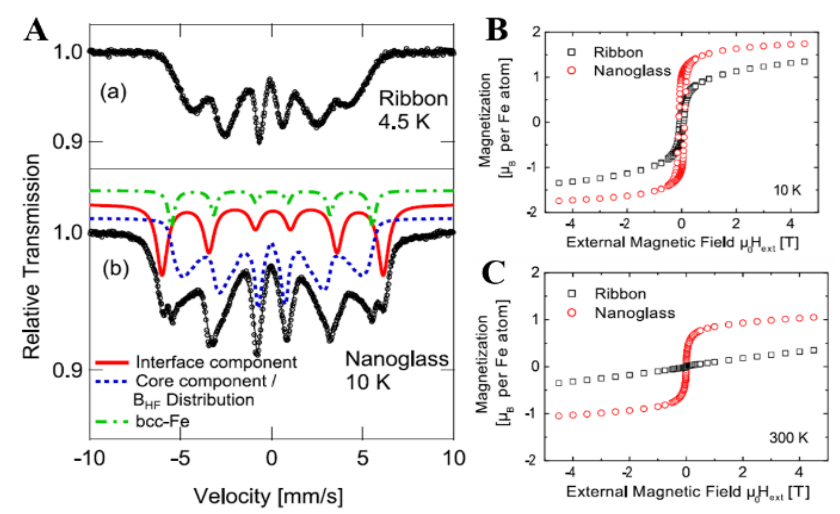

Fig. 6. (A) Low temperature Mossbauer spectra of the (a) melt-spun ribbon and (b) nanoglass. The ribbon is fitted with a unimodal distribution of magnetic hyperfine fields BHF. The nanoglass is fitted with one distribution of BHF (dashed fitting curve), resembling the ribbon spectrum and two additional sextets. (B) Magnetization curves for a nanoglass sample and a melt-spun ribbon at $300 \mathrm{~K}$. The ribbon exhibits a paramagnetic behavior, while the nanoglass shows a ferromagnetic shape reaching a magnetization of $1 \mu \mathrm{B}$ per $\mathrm{Fe}$ atom in the applied magnetic field of $4.5 \mathrm{~T}$. (C) Magnetization measurements at $10 \mathrm{~K}$. The average magnetization increases in the nanoglass to 1.74 (2) $\mu \mathrm{B}$ compared to 1.35 (1) $\mathrm{lB}$ per $\mathrm{Fe}$ atom in the melt-spun ribbon. The error bars are smaller than the symbols. (Reproduced with permission from [11], Copyright $\odot$ 2013, AIP Publishing).

More recently, his group provides a new route for designing high-performance metallic glassy materials by manipulating their atomic structures and helps for understanding the complex atomic structure-property relationship in amorphous materials [12]. The study also indicates that the cryogenic thermal cycling should be considered as the simple and useful way to modify the microstructure and property of metallic glassy materials for engineering applications (Fig. 7).

The recent work of Dr. Gleiter was focused on the polyamorphism. His group also revealed that like the crystalline materials, the properties of amorphous materials can be tailored by tuning the local atomic-to-nanoscale structural configurations [13]. They also provided the experimental evidence of the coexistence of liquid-like and solid-like amorphous phases in a $\mathrm{Ni}_{82} \mathrm{P}_{18}$ amorphous alloy with enhanced thermal stability and plasticity. The manuscript also demonstrated the preparation of $\mathrm{Ni}_{82} \mathrm{P}_{18}$ amorphous alloys using multi-phase pulsed ED within a specific pulse current range. The alloys are consisting of a unique structure factor: for the second peak maximum, there are two sub-peaks with comparable diffraction intensities (Fig. 8). Its consist of the following two kinds of amorphous structural components: nanometer-sized regions with solid-like structure (SLS, the atomic structure of melt-quenched MG-like $\left.\mathrm{Ni}_{82} \mathrm{P}_{18}\right)$ and nanometer-sized regions with liquid-like structure (LLS, of loose packing compared to a melt-quenched Ni-P ribbon sample).

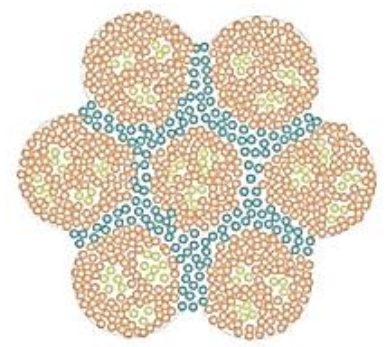

the microstructural model of nanoglasses

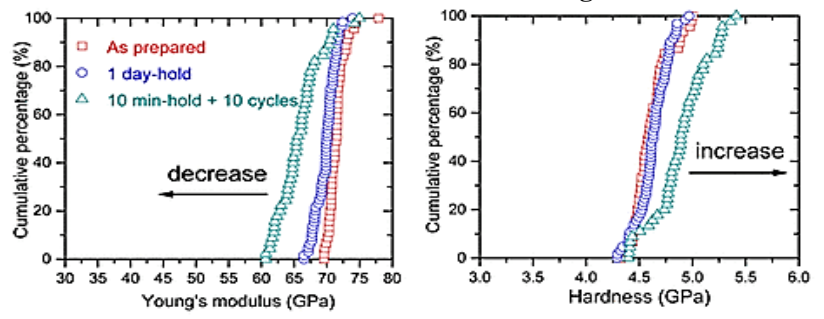

Fig. 7. Microstructural model of the $\mathrm{Sc}_{75} \mathrm{Fe}_{25}$ NGs; Cumulative distributions of (above) Young's modulus and (down) the hardness of the $\mathrm{Sc}_{75} \mathrm{Fe}_{25} \mathrm{NG}$ at the as-prepared state, quenching directly into liquid nitrogen $(77 \mathrm{~K})$ and holding for 1-day state, and cryogenically thermal cycled state. (Reproduced with permission from [12], Copyright (C) 2018, American Chemical Society).
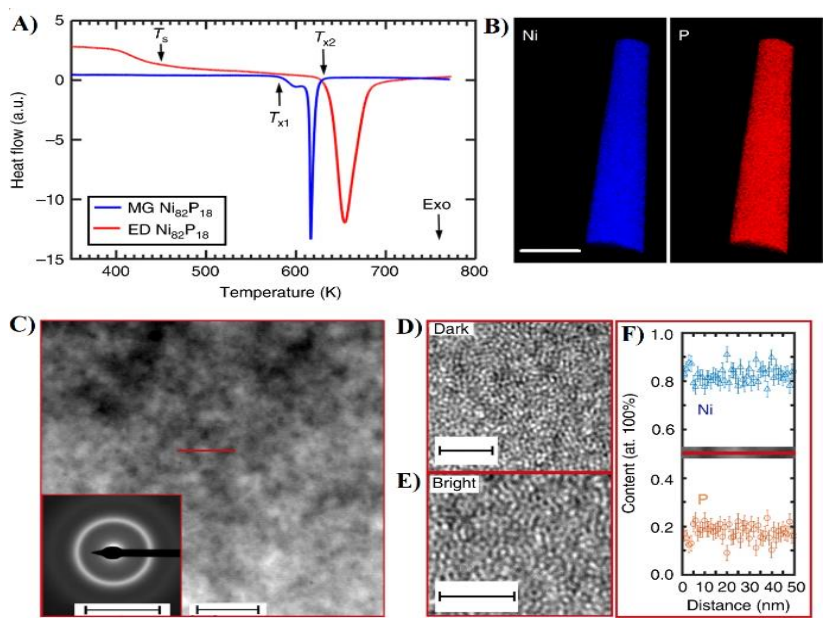

Fig. 8. Thermophysical behaviour and structure inhomogeneity. (a) Calorimetric curve for metallic glass (MG) Ni82P18 ribbon (blue) and electrodeposition (ED) Ni82P18 alloy (red). (b) Composition mapping by $3 \mathrm{D}$ atomic probe, showing homogeneous $\mathrm{Ni}$ and $\mathrm{P}$ element distribution at nanoscale in ED Ni82P18 alloy; scale bar, $100 \mathrm{~nm}$. (c) High angle annular dark field image (HAADF) of the ED Ni82P18 alloy, showing bright/dark contrast at $10-20 \mathrm{~nm}$; scale bar, $50 \mathrm{~nm}$. The inset is the selective area electron diffraction pattern (SAED) with diffused halos, illustrating the amorphous nature of the as-prepared ED Ni82P18 alloy; scale bar, $10 \mathrm{~nm}-1$. High-resolution transmission electron microscopy (HRTEM) images with the typical maze-like amorphous structure for the dark region (d) and bright region (e). HAADF image; scale bars, $2 \mathrm{~nm}$. (f) Energydispersive X-ray spectroscopy (EDS) line mapping results for Ni element and $\mathrm{P}$ element, showing that there is no obvious composition fluctuation. The average composition is $\mathrm{Ni} \sim 82$ at. $\%$ and $\mathrm{P} \sim 18$ at. $\%$. The mapping line is indicated by the red line as shown in (c). Error bars are defined as the standard deviation (Reproduced with permission from [13], Copyright $($ ) 2019, Springer Nature). 


\section{Advanced Materials Letters www. vbripress.com/aml}

\section{Awards and recognitions}

Because of his ground-breaking work and research in nanoscience and nanotechnology, Prof. Herbert Gleiter has received a plethora of awards, accolades, and honours in his illustrious career. Some of the most esteemed awards that he has received over the years are: Leibniz Prize of the German National Science Foundation (1988), Max-PlanckResearch Award of the Humboldt Foundation and the Max Planck-Society (1993), the Acta Materialia Gold Medal of the Federation of European Material Societies, Edward DeMille Campbell Award, American Society for Metals (2012), Staudinger-Durrer Award of the Swiss Federal Institute of Technology (2008), the Blaise Pascal Medal of the European Academy of Sciences, Belgium (2009), Nanomaterials Award (2012), the Cothenius Medal of the German National Academy of Sciences Leopoldina (2015) and Advanced Materials Laureate (2019) [1, 14].

Besides these prestigious honours, he has received honorary doctorates, fellowships, and professorships from the best universities of the world and he has been elected as an honorary member or member in many national academies and professional committees all around the world, like the American Academy of Arts and Sciences, German National Academy of Sciences, US Academy of Engineering, Materials Research Society of the United States, the Japanese Society for the Promotion of Science, Research Society of India, of the United States, the American Nano Society, and many more [2].

\section{Global Co-operative networking}

Herbert Gleiter's co-operations with external European partners involve predominantly colleagues from institutions located in the UK. In the field of intercrystalline interfaces, the contributions of the following partners were important. Two research projects on grain boundaries were carried out jointly with Robert Cahn (University of Sussex) between 1972 and 1975. Moreover, Robert was a member of the Advisory Board of the Institute of New Materials at Saarbruecken and Herbert Gleiter was one of the co-authors of Cahn-Haasen's textbook on Physical Metallurgy. Helmut Mykura (University of Warwick) spent in 1979 a sabbatical leave at Saarbruecken working on new methods for studying the atomic structure and the energy of inter-crystalline interfaces. This work resulted in three joint publications and provided the basis for the co-operation between Mykura's and Gleiter's group during the subsequent years. Peter Pumphrey spent three years at Saarbruecken as a Humboldt Scholar. After returning to the Central Electricity Research Laboratory at Leatherhead, he co-operated with the group at Saarbruecken for about three more years on the interaction of lattice defects with grain boundaries. These studies resulted in nine joint publications. Stuart Campbell (today at the Australian National University, ANU, at Canberra) stayed with Uli Gonser and Herbert Gleiter as a Humboldt Scholar at Saarbruecken. He studied - by means of Moessbauer spectroscopy - the atomic structure of nanocrystalline metals. This work resulted in four joint publications and it was continued when Herbert Gleiter spent a sabbatical leave at ANU in 1985. During Gleiter's time at Harvard University, he co-operated with Mike Ashby (today at Cambridge University). Mike is a coauthor of the first publication utilizing molecular dynamics to study the atomic structure of grain boundaries. The results obtained resulted in the "structural unit model" of boundaries that provides the basis for all of today's models. In the field of polymeric materials, Mervin Miles (University of Bristol) spent about four years at Saarbruecken working on the molecular structure and the processes involved in the plastic deformation of semicrystalline polymers.

The co-operations with colleagues from outside of Europe involve primarily groups in the US, China and India. For a period of about eight years (beginning in 1993) Dieter Wolf and Herbert Gleiter headed a research group at Argonne National Laboratories (ANL). This group was initiated when both were awarded the Max Planck Research Prize funding this group for three years. The funding in the subsequent five years was primarily obtained from NSF. The idea of this group was to combine the expertise in molecular dynamics at ANL with the expertise at Saarbruecken in the field of nanocrystalline materials in order to study the atomic structure and thermal stability of these materials. Five members of this group were offered professorships when the group was closed down in 2001 and the results obtained (published in the form of 14 papers) are cited today about 3500 times. The second co-operation outside of Europe was "catalyzed" by Gleiter's role as a foreign member of the National Academies of the US (NAE), India (INSA and INAE) and previously China (CAS) as well as a member of the German National Academy (Leopoldina). His role in the CAS and in the Leopoldina turned out to be crucial for starting up a new research institute similar to a Max Planck Institute in Germany) working on nanoscience at Nanjing (named "Herbert Gleiter Institute of Nanoscience"). The institute directors are Profs. Ke Lu and J. Xin (from the CAS). The partners from the Leopoldina are Profs. H. Fuchs, H. Hahn and $\mathrm{H}$. Gleiter. Similarly, as a foreign member of the Indian National Academies (INSA and INAE) and as a member of the Leopoldina, Gleiter was able to initiate several new research programs between Germany and the IIT Kharagpur, the IISc Bangalore and the Universities of Chennai as well as Hyderabad.

\section{Contributions to the society}

The contributions of Prof. Gleiter to the society may be divided in two portions: (1) contributions resulting from Prof. Gleiter's work to today's as well as to tomorrow's economies. (2) Contributions by initiating new intellectual structures.

(1) Several comprehensive summaries outlining the contributions made by the invention of nano-materials to today's economy may be found in the Nanotech Magazine and they have been recently evaluated in a report by the 


\section{Advanced Materials Letters www. vbripress.com/aml}

German Government. According to this report, the annual value of the products based on nano-materials is beyond 2 billion US\$ with a growth rate of about 15 to $20 \%$ per year. A comparable evaluation was performed 3 years ago by the Chinese Academy of Sciences. In this evaluation, the Chinese Academy of Sciences (CAS) selected nanomaterials to be the most promising contribution in the field of modern materials with the potential of opening the way to new technologies. In fact, it was recommended - as was mentioned above - to start up (at Nanjing University of Science and Technology) a new research institute on nanoscience (named "Herbert Gleiter Institute of Nanoscience, HGI)" (Fig. 9, 10). The goal of this institute is to perform original research by providing research conditions comparable to the ones at elite institutes e.g. in Europe, in Japan or in the US and totransfer some of the results obtained into commercial products.

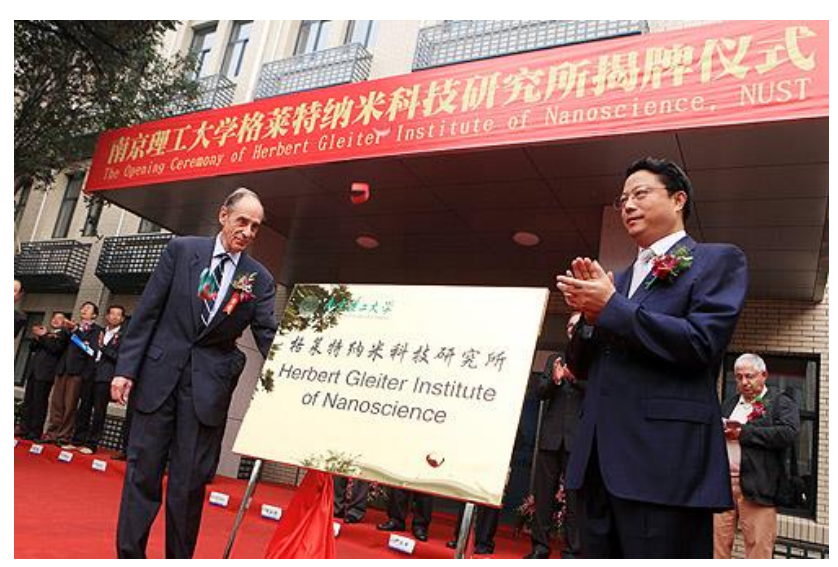

Fig. 9. Prof. Herbert Gleiter during the opening ceremony of Herbert Gleiter Institute of Nanoscience, China.

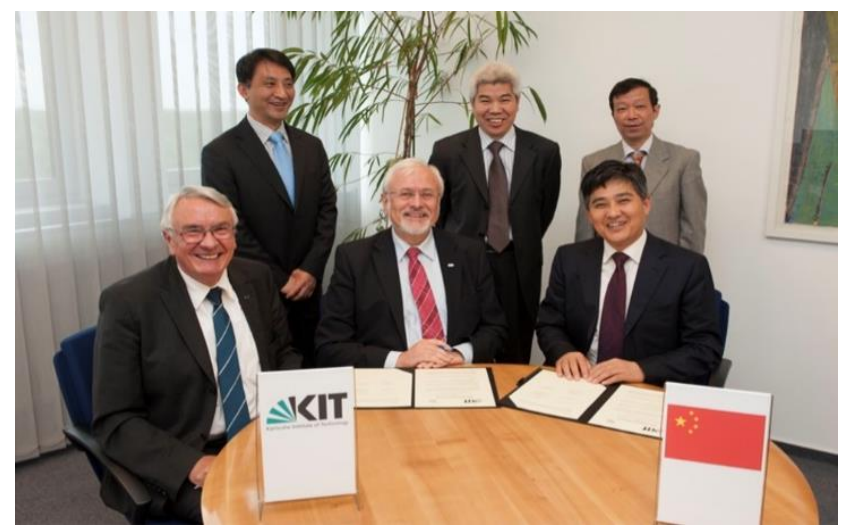

Fig. 10. Visit of the delegation of NJUST to KIT on June 15, 2012 for signing the memorandum of understanding between NJUST and KIT. In photo, from NJUST: Prof. L. Quian (vice President), Prof. J. Wang (Deputy Dean. HGI), Prof. H. Wang (Project Manager); KIT: Prof. E. Umbach (President) and Prof. D. Loehe (Vice President).

The selection of this goal should be seen in line with the performance of two research institutes that have been initiated by Prof. Gleiter in 1984 and 2004. In 1984 the Government of the State of Saarland (Germany) founded the Institute of New Materials, INM (initiated and directed initially by Prof. Gleiter) at the University of the Saarland.
This foundation was motivated by the attractive technological perspectives of nano-crystalline materials that were discovered in 1979 by Prof. Gleiter's group at the University of the Saarland. The contribution of the INM to the society may be characterized by a few numbers: 34 start-up companies were founded by members of this institute using their research results obtained at the INM. These companies created more than 1800 jobs. Moreover, 8 of the former INM members have been appointed to professorships. In 2004 the economic perspectives of nanotechnology, in particular nanomaterials motivated the Karlsruhe Institute of Technology (KIT) to found an Institute on Nanotechnology (INT) (initially directed by Profs. Fenske, Gleiter and Lehn). An evaluation in 2012 rated the INT on the ranks 1 and 3 as far as its contributions to basic research and to the economy of Germany are concerned. In fact, based on the work of the INT, the KIT was appointed recently to become the Germany's center of energy research and technology.

Concerning the contributions of Prof Gleiter to tomorrow's economy, nano-glasses seem to have the potential to coin tomorrow's society by opening fundamentally new economic and technological perspectives. As was pointed out above, nano-glasses are non-crystalline materials with a microstructure that corresponds to the microstructure of single or multi-phase poly-crystalline materials, such as steels, ceramics etc. which provide the basis of today's technology. This analogy between the microstructures of today's crystalline materials and of nano-glasses suggest the following spectacular long-term contribution of nano-glasses to tomorrow's technology. The utilization of the new, partially spectacular properties of nano-glasses (pointed out above) may open in the foreseeable future the door to a new age of technologies, a kind of "glass age". The new technologies of this "glass age" would be based on the new properties of nano-glasses. In other words, the "glass age" would be historically in line e.g. with the bronze age or the iron age that were based on the new properties of bronze or iron when they were discovered.

(2) The original structure and mission (indicated briefly above) of the HGI at Nanjing has been recently reoriented by him into an "elite institute" (deviating from some Chinese traditions). The motivation for this reorientation was Prof Gleiter's vision to broaden the contribution of the HGI to the society. With its new structure, the HGI cannot only provide outstanding research conditions (e.g. in terms of equipment, funding, salaries etc.) but also create an intellectual institute structure that is comparable to the ones in elite institutes e.g. in the US, in Japan or in Europe.

In other words, the HGI will not only be able to provide outstanding young scientists with excellent working conditions but it will also stimulate a new intellectual research atmosphere. In that sense the re-orientation of the HGI is likely to contribute - in the long run - to a "reshaping" of the research and educational "landscape" of China by pioneering the idea and necessity of structures that 


\section{Advanced Materials Letters www. vbripress.com/aml}

deviate from some of the Chinese traditions but promote new ideas and structures that will provide attractive opportunities in particular for young scientists and engineers.
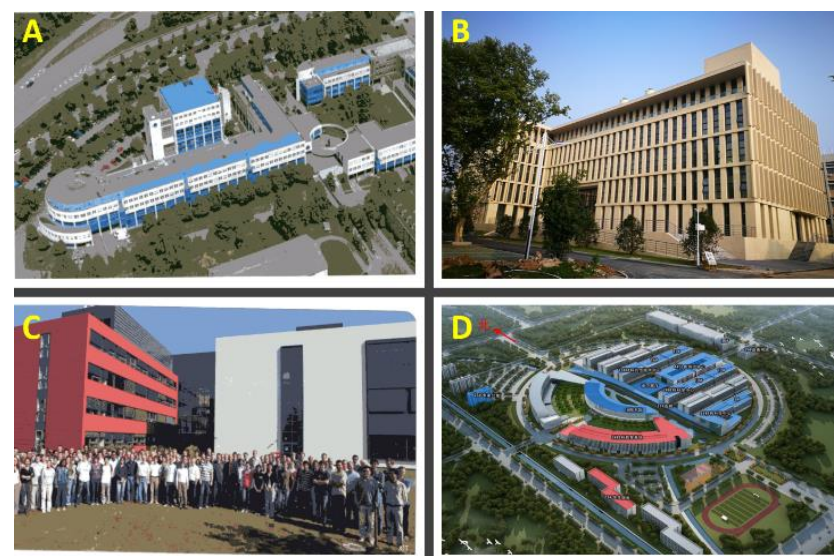

Fig. 11. Photo of the institutes and centres founded by Prof. Herbert Gleiter. (A) Leibniz Institute of New Materials, Saarbruecken; (B) Herbert Gleiter International Laboratory; (C) Institute of Nanotechnology, Kalsruhe; (D) Herbert Gleiter Institute of Nanosciences

The institute was established to produce cutting-edge research in the field of nanotechnology and nanoscience that can eventually lead to real-world applications. The institute also fosters the careers of many young academics and researchers from all over the world. It helps the young researchers and gives them a lot of opportunities to maximize their potential. Over the years, Institute of Nanotechnology has grown both in terms of prestige and numbers. Today, it is recognized as a prime organization working for the development of nanoscience and nanotechnology.

Recently, to promote the research and innovation in China, he is establishing another centre, i.e., Herbert Gleiter International Laboratory, that is presently under construction and that will be one of the research centers of the Chinese Academy of Sciences at Shenyang. Advanced Materials Letters feels very honored and privileged to confer upon him the 'Researcher of the Year 2020' for his several years of diligence and uncountable efforts for the promotion of advanced nanomaterials. International Association of Advanced Materials heartily congratulate Prof. Herbert Gleiter for being recognized as the 'Researcher of the year 2020'.

\section{References}

1. "Report of the Asian Advanced Materials Congress - 2019, Singapore”, blog post 21 November 2019, viewed 08 January 2020, $<$ https://www.iaamonline.org/blog/asian-advanced-materialscongress-2019/>.

2. "Advanced Materials Laureate 2019: Professor Herbert Gleiter - one of the highest awards in the field of Advanced Materials", post 21 November 2019, viewed 08 January 2020,

$<$ https://news.cision.com/iaam/r/advanced-materials-laureate-2019-professor-herbert-gleiter---one-of-the-highest-awards-in-the-field, c2975167>.
3. "Advanced Materials Laureate", International Association of Advanced Materials, viewed 08 January 2020,

$<$ https://www.iaamonline.org/awards/advanced-materialslaureate>.

4. "The Story of IAAM - A Journey to Achieving Global Excellence", blog post 18 August 2019, viewed 08 January 2020,

<https://www.iaamonline.org/blog/the-story-of-iaam-a-journey-toachieving-global-excellence/>.

5. "CV of Professor Herbert Gleiter" viewed 08 January 2020, $<$ https://www.leopoldina.org/fileadmin/redaktion/Mitglieder/CV_G leiter_Herbert_EN.pdf $>$.

6. "Honorary Doctor of Science Professor Herbert GLEITER", viewed 08 January 2020,

<https://www.cityu.edu.hk/sites/g/files/asqsls3821/files/201906/e_Gleiter.pdf $>$.

7. Karch, J.; Birringer, R.; Gleiter, H.; Nature; 1989, 330, 556.

8. Jing, J.; Kramer, A.; Birringer, R.; Gleiter, H.; Gonser, U.; Journal of Non-Crystalline Solids, 1989, 113, 167.

9. Gleiter, H.; Acta Materialia; 2000, 48, 1.

10. Weissmuller, J.; Viswanath, R. N.; Kramer, D.; Zimmer, P.; Wu"rschum, R.; Gleiter, H.; Science, 2003, 300.

11. Witte, R.; Feng, T.; Fang, J.X.; Fischer, A.; Ghafari, M.; Kruk, R.; Brand, R.; Wang, D.; Hahn, H.; Gleiter, H.; Applied Physics Letters; 2013, 103, 073106, 1

12. Wei-Hong Liu; Sun, B. A.; Gleiter, Herbert; Lan, Si; Tong, Yang; Xun-Li Wang, Hahn, Horst; Yang, Yong; Kai, Ji-Jung; Liu, C. T.; Nano Lett.; 2018, 18, 7, 4188.

13. Lan, Si; Guo, Chunyu; Zhou, Wenzhao; Ren, Yang; Almer, Jon; Pei, Chaoqun; Hahn, Horst; Chain-Tsuan Liu, Feng, Tao; Wang, Xun-Li; Gleiter, Herbert; Commun. Phys.; 2019, 2, 117.

14. "Professor Herbert Gleiter" viewed 08 January 2020, <https://www.int.kit.edu/staff_herbert.gleiter.php〉. 